\title{
THE NEW 250kV SINGLE STAGE AMS SYSTEM AT CAIS, UNIVERSITY OF GEORGIA: PERFORMANCE COMPARISON WITH A 500kV COMPACT TANDEM MACHINE
}

\author{
G V Ravi Prasad ${ }^{1}$ John E Noakes • Alexander Cherkinsky • Randy Culp • Doug Dvoracek \\ Center for Applied Isotope Studies, University of Georgia, Athens, Georgia 30602, USA.
}

ABSTRACT. A new $250 \mathrm{kV}$ single stage AMS accelerator (SSAMS) was installed at the Center for Applied Isotope Studies, University of Georgia. The accelerator is intended to be used primarily for radiocarbon measurements of natural and biobased samples, while all other samples such as marine, geological, atmospheric and archaeological samples are measured on the decade-old $500 \mathrm{kV}$ compact tandem accelerator (CAMS). The new AMS system is equipped with a 134-cathode MCSNICS ion source. In this article, we show the results of the tests carried out on standards and blanks and compare the performance of the new machine with that of the CAMS unit. We have also compared the stable isotope data from AMS measurements to the conventional isotope ratio mass spectrometers (IRMS) data.

\section{INTRODUCTION}

Radiocarbon dating has seen an enormous growth in the last decade and most of it can be attributed to the availability of dedicated compact accelerators custom built for ${ }^{14} \mathrm{C}$ dating. The Center for Applied Isotope Studies at the University of Georgia installed a 500kV compact tandem accelerator (CAMS) in 2000, which is one of the earliest compact machines built by the National Electrostatics Corporation. This is a conventional tandem accelerator consisting of a charging chain encased in a vessel pressurized with $\mathrm{SF}_{6}$ gas. The unit is equipped with a 134-cathode MC-SNICS ion source. Since 2000, CAIS has been providing ${ }^{14} \mathrm{C}$ AMS dates to various research units both in academic and commercial establishments (Roberts et al. 2004; Cherkinsky et al. 2010). The sample throughput has steadily grown over the years. After a decade of operation, we have encountered a series of breakdowns due to wornout components in the accelerator tank. We have replaced many of the moving parts such as the charging chain, generating voltmeter (GVM), bearings of the chain motor and the shaft motor, and sheaves of the charging pulleys just in the last $2 \mathrm{yr}$.

As CAIS analyzes a large number of natural products and biobased samples that are mostly submitted by the industry, we decided to buy and install a second AMS unit to keep up with the sample flow. The $250 \mathrm{kV}$ single stage AMS (SSAMS) unit made by NEC seemed to be the right choice to complement the existing $500 \mathrm{kV}$ unit due to the following reasons. The natural and biobased samples are measured for their ${ }^{14} \mathrm{C}$ concentration with reported precisions no better than $1 \%$, so there are less stringent requirements on the data precision and background level. Therefore, the lower beam transmission and lower beam energy of SSAMS are not an issue with these samples. The SSAMS unit is air-insulated and there are no moving parts or pressurized tank around the high-voltage section. Considering the aforementioned issues with the pelletron accelerator, SSAMS offers simplicity with regard to the accelerator section of the instrument, as it does not require regular maintenance. Negative ions are stripped off after accelerating to the deck potential and there is no second stage acceleration of positive ions. However, CAMS being a tandem machine produces higher beam energy leading to higher transmission of ions with +1 charge state in comparison. The SSAMS unit was installed in November 2011. The schematic of both AMS units is shown in Figure 1.

\footnotetext{
${ }^{1}$ Corresponding author. Email: gvrp@uga.edu.
} 
GVR Prasad et al.
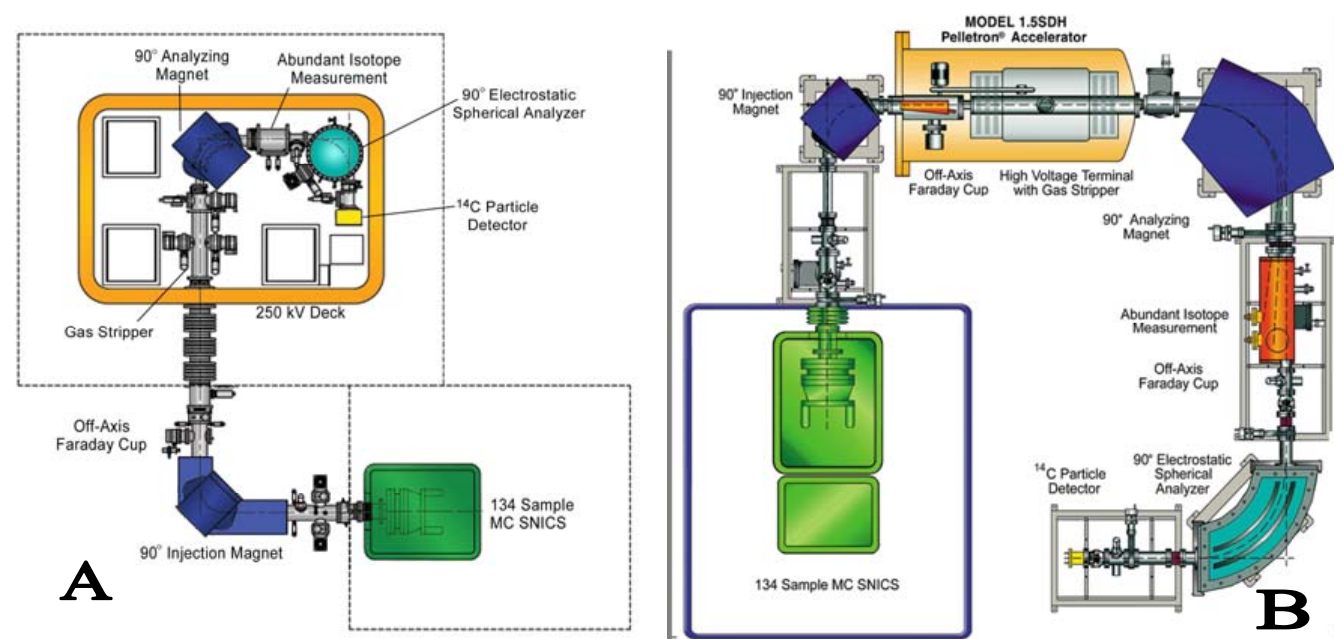

Figure 1 Schematic of the 2 AMS units: A) 250kV SSAMS and B) 500kV CAMS. Figures provided by National Electrostatics Corporation.

The 134-cathode MC-SNICS ion source on the $500 \mathrm{kV}$ unit has been performing satisfactorily, so we opted for the same 134-cathode ion source for the SSAMS. The acquisition software allows running the samples in groups, so drifts in tuning parameters is not an issue even with a full cathode wheel. The closed-cycle cathode cooling system on CAMS is connected to a water-cooled heat exchanger to remove the heat, whereas on the SSAMS system an air-cooled heat exchanger performs the task and yet appears adequate. The new ion source also allows for cathode position adjustment if needed, even while in operation. The injection beam line is equipped with 2 Varian turbomolecular pumps, each having a pumping speed of $250 \mathrm{~L} / \mathrm{s}$. A beam profile monitor and Y-steerers are also provided in the source beam line.

The SSAMS unit occupies nearly equal floor space as the CAMS unit. Two TDK-Lambda power supplies of $1.5 \mathrm{~kW}$ and $2.4 \mathrm{~kW}$ power the injection and analyzing magnets, respectively. These power supplies are air-cooled and extremely compact, and provide good magnetic field stability when controlled through the Hall probe feedback loop. In comparison, the Danfysik magnet power supplies on the CAMS unit are bulky and water-cooled, but maintain excellent current (and therefore magnetic field) stability.

The einzel lens situated between the injection magnet and the accelerator tube allows for additional beam focusing. In contrast, the CAMS unit does not have an einzel lens in its pre-acceleration stage. The post-acceleration stage includes the analyzing magnet, electrostatic spherical analyzer (ESA), and the detector. The entire stage housing all the electrical and vacuum units and the electronics floats at the deck voltage. This stage is enclosed in a protective cage and is interlocked for safety.

The SSAMS control and data acquisition is handled by NEC's proprietary ACT hardware interface running a Linux operating system. There are $2 \mathrm{ACT}$ computers, one situated near the injection magnet and the second on the high-voltage stage, and both are connected to the main computer that is running the Scientific Linux operating system. On the 500kV unit, CAMAC hardware controls the accelerator and data acquisition. AccelNet software provides the control and data acquisition interface for AMS measurements on both machines. The strip-chart feature of this software allows monitoring of up to 24 parameters for up to $120 \mathrm{hr}$. Off-axis ${ }^{12} \mathrm{C}^{+}$and ${ }^{13} \mathrm{C}^{+}$ion currents are fed to the cur- 
rent integrator and the ${ }^{14} \mathrm{C}^{+}$ions are measured using a solid state particle detector. The data acquisition system is based on National Instruments' PC-based PXI platform.

\section{THE AMS METHOD}

The ion source parameters are set to produce a beam of negative ions of $45 \mathrm{keV}$ energy. The extracted ions are not necessarily "atomic" ions, but there are also a large number of molecular ion species. The mass distribution of the ions extracted from a carbon cathode that are leaving the ion source ranges from 1 to well over 100 (Middleton 1990). ${ }^{12} \mathrm{C}^{-}$contributes the bulk of the ion current, whereas hydrogen and oxygen are the ubiquitous contaminants in any cathode material, and higher masses are usually clusters of atoms. The injection magnet can be tuned to select a specific mass (strictly the momentum) for analysis. A voltage bouncer controls sequential injection of ${ }^{12} \mathrm{C}^{-},{ }^{13} \mathrm{C}^{-}$, and ${ }^{14} \mathrm{C}^{-}$through the injection magnet by applying voltage pulses on the insulated chamber. The negative ions are accelerated to the set potential where they undergo charge exchange collisions with argon gas on exiting the accelerating section. The analyzing magnet separates the accelerated ions based on their momentum. ${ }^{12} \mathrm{C}^{+}$and ${ }^{13} \mathrm{C}^{+}$particle currents are measured in off-axis Faraday cups. The ${ }^{14} \mathrm{C}^{+}$ions are made to pass through the ESA, which acts as the energy filter. An electrostatic deflector situated between the ESA and the detector is synchronized with the voltage bouncer to protect the detector by blocking the access during non- $-{ }^{14} \mathrm{C}^{+}$parts of the bounce cycle.

Table 1 General characteristics and operational values of both AMS units during the 6-month period mentioned in the text.

\begin{tabular}{lll}
\hline & SSAMS & CAMS \\
\hline Operating terminal voltage & $240 \mathrm{kV}$ & $470 \mathrm{kV}$ \\
Acceleration & Single stage & Tandem \\
Terminal stability & $\pm 20 \mathrm{~V}$ & $\pm 200 \mathrm{~V}$ \\
Total beam energy & $285 \mathrm{keV}$ & $994 \mathrm{keV}$ \\
Beam transmission & $30 \%$ & $40 \%$ \\
Ion source & $134-$ cathode MC-SNICS & $134-$-cathode MC-SNICS \\
${ }^{12} \mathrm{C}^{-}$source currents & $70-100 \mu \mathrm{A}$ & $80-110 \mu \mathrm{A}$ \\
Beam energy from source & $45 \mathrm{keV}$ & $54 \mathrm{keV}$ \\
Alfa Aesar graphite & $\sim 53 \mathrm{ka}$ & $\sim 59 \mathrm{ka}$ \\
AMS precision (modern sample) & $\pm 0.36 \mathrm{pMC}$ & $\pm 0.30 \mathrm{pMC}$ \\
\hline
\end{tabular}

\section{RESULTS AND DISCUSSION}

Natural and biobased samples are mostly analyzed on the SSAMS unit, whereas the geological and other older samples are run on the CAMS unit. We use the NIST OX-I as our primary standard in all measurements. The data of OXII standards and blanks measured during the period January-June 2012 are selected for comparison. The general characteristics of both machines are presented in Table 1. The data of 34 OXII measurements on SSAMS and 19 OXII measurements on CAMS are presented in Figure 2. The mean values of OXII measurements show excellent agreement with each other and very close to the reference value of 134.066 . However, the overall $1 \sigma$ deviation as seen from Figure 2 is much larger than the typical measured AMS precisions. The data reflects the overall variability in sample preparation and AMS measurement over a 6-month period. However, Figure 2 does not exclude the data points that are due to occasional issues with sample preparation and machine stability, and contribute to the larger scatter. The total uncertainty of measurement is best represented by a lab multiplier, which is the ratio of long-term variability to the precision from a single measurement. We estimated the lab multiplier from a much larger pool of data of OX1, which is 


\section{GVR Prasad et al.}

our primary standard. Every sample group has at least $2 \mathrm{OX} 1 \mathrm{~s}$ for normalization. For the purpose of estimating the lab multiplier, we have treated one of the OX1s as unknown. The data over a period of $1 \mathrm{yr}$ show a $1 \sigma$ deviation of $0.42 \mathrm{pMC}$ on CAMS and 0.52 on SSAMS. Based on the quoted precisions of Table 1, the lab multiplier is about 1.4 for both machines.
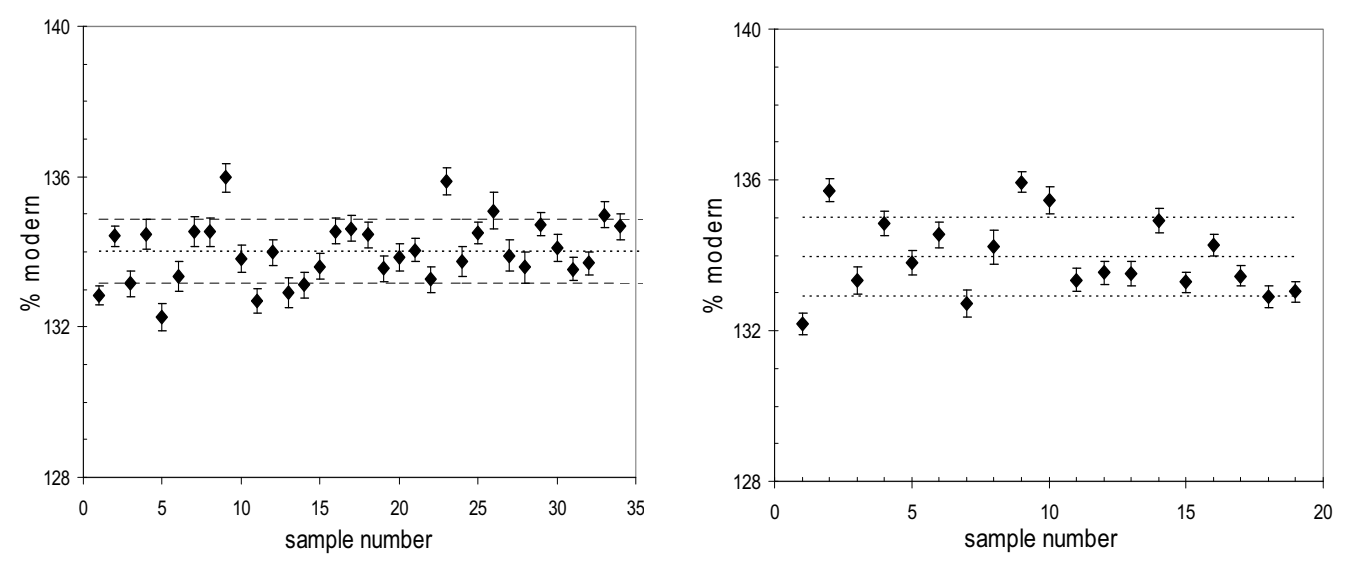

Figure 2 OX-II data from both machines are plotted for comparison. The data from SSAMS (on the left) show a mean value of $134.02 \pm 0.84 \mathrm{pMC}$. The data from CAMS (on the right) show a mean value of $133.95 \pm 1.05 \mathrm{pMC}$. The mean values and their standard deviations are shown by dashed lines. See the text for additional information.

The data of blanks are plotted in Figure 3. We have shown the numbers from unprocessed Alfa Aesar graphite as well as processed blanks. The graphite line for processing natural products is separate from the line that processes geological and other samples. Blank1 refers to the blank prepared on the graphite line for natural products. Blank2 refers to the other ${ }^{14} \mathrm{C}$ sample preparation line. In both cases, blanks are prepared from anthracite. Obviously, the tandem accelerator is superior to the single stage accelerator with regard to blanks. The higher particle energy reduces the survival probability of the molecular ions in the stripping column, while enhancing mass separation. The mean apparent age of 53k from Alfa Aesar graphite is still impressive for the single stage machine, which has shown great improvement in lowering the background since the first built single stage system (Skog et al. 2010). We hypothesize this could be due to the presence of an additional turbomolecular pump near the stripper.

During this period, we have also compared the stable isotope ratio $\delta^{13} \mathrm{C}$. The AMS accelerators are not primarily intended for $\delta^{13} \mathrm{C}$ measurements, and can never approach the precisions provided by the conventional isotope ratio mass spectrometers (IRMS), which are more precise by 2 orders of magnitude. Currently, we use the IRMS data to make the fractionation correction to the measured ${ }^{14} \mathrm{C} /{ }^{13} \mathrm{C}$ ratios. It is still important to determine the $\delta^{13} \mathrm{C}$ values using AMS to optimize the operating parameters and to minimize the deviation from IRMS data. In Figure 4, we compare the $\delta^{13} \mathrm{C}$ values from AMS measurements to the conventional IRMS values. The data from SSAMS shows a sharper profile and in much better agreement with the IRMS data as compared to the CAMS data. Though terminal potential fluctuations of SSAMS unit are only $\sim 20 \mathrm{~V}$ as opposed to $\sim 200 \mathrm{~V}$ on the tandem accelerator, we believe there are other contributing factors that significantly affect the measured $\delta^{13} \mathrm{C}$ values. We have observed a significant reduction in ${ }^{12} \mathrm{C}$ beam transmission with the increase in beam current, but ${ }^{14} \mathrm{C} /{ }^{13} \mathrm{C}$ ratios are unaffected because of the smaller ${ }^{13} \mathrm{C}$ beam currents. The transmission loss is believed to be due to space charge at higher current coupled with scattering in the stripper canal (J B Schroeder, private communication, 2012). These observations are in agreement 

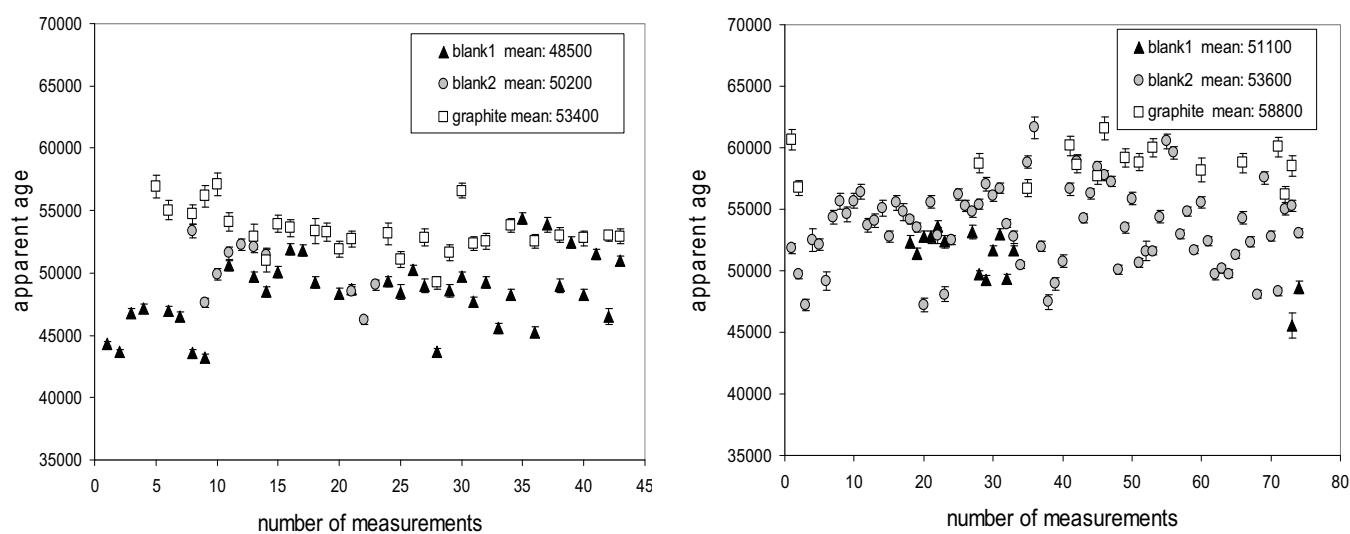

Figure 3 Data of blanks measured on SSAMS on the left, and CAMS on the right. Blank1 and blank2 are prepared on 2 different sample preparation lines (see text). The data of unprocessed Alfa Aesar graphite is also shown in the figure.

with earlier reported data (Skog et al. 2010). This effect is more pronounced on the $250 \mathrm{kV}$ unit but less so on the $500 \mathrm{kV}$ unit. As a consequence of beam current affecting the transmission, the operating parameters for a better $\delta^{13} \mathrm{C}$ measurement differ somewhat from the parameters to make the best ${ }^{14} \mathrm{C} /{ }^{13} \mathrm{C}$ measurement. As we are more interested in the ${ }^{14} \mathrm{C}$ data, some compromise on the $\delta^{13} \mathrm{C}$ precision is accepted. Owing to the strong dependence of beam transmission on ${ }^{12} \mathrm{C}^{-}$beam current, we do not use the AMS $\delta^{13} \mathrm{C}$ values for stable isotope correction at this point of time. It is possible to correct for the transmission dependence and we are studying this behavior for different conditions. We are currently doing a detailed investigation on the isotopic fractionation effects on both machines and the results will be published elsewhere.

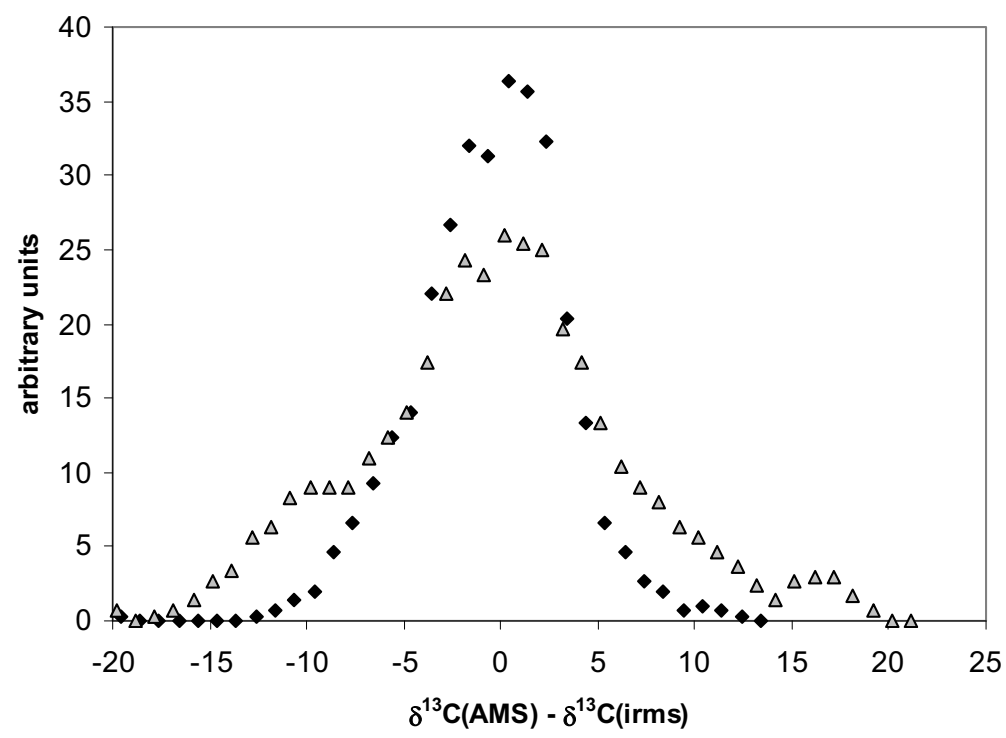

Figure $4 \delta^{13} \mathrm{C}$ measured from AMS are compared to the values obtained from the conventional IRMS values. SSAMS data are shown as solid diamonds and CAMS data are shown as triangles. 


\section{CONCLUSIONS}

The new SSAMS unit is mostly being used for ${ }^{14} \mathrm{C}$ measurements of natural and biobased samples. We compare the performance of both AMS units during January-June 2012. Data are compared for OX-II (check standard) and blanks. The OX-II measurements from multiple cathodes produced consistent data on both machines. The blanks on SSAMS are slightly poorer compared to the CAMS unit, but considering that the particle energy is only a third of the particle energy from the CAMS unit, the numbers are still impressive, and far superior to the first single stage AMS system built for Lund University. The beam transmissions are nearly $30 \%$ and $40 \%$ for the single stage and the tandem machines, respectively, and hence the data precision for the same counting time is better on CAMS. Stable isotope ratio values of $\delta^{13} \mathrm{C}$ from both the AMS machines are compared to the values from the conventional isotope ratio mass spectrometer. SSAMS measurements are superior to CAMS in this case. Better terminal stability of SSAMS unit is an obvious factor, but we are also investigating other sources that contribute to the fractionation effects.

\section{REFERENCES}

Cherkinsky AE, Culp RA, Dvoracek DK, Noakes JE. 2010. Status of the AMS facility at the Center for Applied Isotope Studies, University of Georgia. Nuclear Instruments and Methods in Physics Research B 268(7-8):867-70.

Middleton R. 1990. A Negative Ion Cook Book. Philadelphia: University of Pennsylvania.

Roberts ML, Culp RA, Dvoracek DK, Hodgins GWL,
Neary MP, Noakes JE. 2004. The ${ }^{14} \mathrm{C}$ AMS system at the University of Georgia. Nuclear Instruments and Methods in Physics Research B 223-224:1-4.

Skog G, Rundgren M, Sköld P. 2010. Status of the single stage AMS machine at Lund University after 4 years of operation. Nuclear Instruments and Methods in Physics Research B 268(7-8):895-7. 